\title{
Charm Spectroscopy at BABAR
}

\section{Denis Altenburg*}

University of Dortmund, Germany

E-mail: d.altenburg@physik.tu-dresden. de

In this note we present results on charmed hadron decays recently obtained from data recorded with the BABAR detector at the Stanford Linear Accelerator Center (SLAC) PEP-II B-Factory.

1. A Dalitz plot analysis of the three body decay $D^{0} \rightarrow \bar{K}^{0} K^{+} K^{-}$has been performed and the branching ratio of $D^{0} \rightarrow \bar{K}^{0} K^{+} K^{-}$and $D^{0} \rightarrow \bar{K}^{0} \pi^{+} \pi^{-}$has been measured very accurately to be

$$
B R=\frac{\Gamma\left(D^{0} \rightarrow \bar{K}^{0} K^{+} K^{-}\right)}{\Gamma\left(D^{0} \rightarrow \bar{K}^{0} \pi^{+} \pi^{-}\right)}=(15.8 \pm 0.1(\text { stat. }) \pm 0.5(\text { syst. })) \times 10^{-2}
$$

Moreover a partial wave anaylsis has been done in order to separate the S- and P-wave contributions. As a result the $a_{0}(980) \rightarrow K K$ lineshape has been extracted for the first time almost background free and we further found a very clean S-P-interference within the $\phi(1020)$ mass region.

2. The mass of the $\Lambda_{c}^{+}$baryon has been obtained from the low- $Q$-value modes $\Lambda_{c}^{+} \rightarrow$ $\Sigma^{0} K_{s}^{0} K^{+}$and $\Lambda_{c}^{+} \rightarrow \Lambda K_{s}^{0} K^{+}$in order to minimize systematic uncertainties mainly due to detector effects. We determined

$$
m_{\Lambda_{c}^{+}}=(2286.46 \pm 0.14) \mathrm{MeV} / c^{2}
$$

3. A study of $\Omega_{c}^{0}$ production in continuum and $\Upsilon(4 S)$ events showed up a very clear first evidence for $\Omega_{c}^{0}$ production in $B$ meson decays.

International Europhysics Conference on High Energy Physics

July 21st - 27th 2005

Lisboa, Portugal

\footnotetext{
* Speaker.
} 


\section{Introduction}

Although the $B A B A R$ project is known as a $B$ meson factory there is much more than $B$ physics which can be done at this facility. The data sample recorded by the BABAR detector since October 1999 contains a large amount of charmed-hadron decays, which can be used to perform precision measurements on the charm sector.

\section{The BABAR Detector}

The following is a brief summary of the most important components for the 3 analyses presented here, which are the tracking devices and the particle identification system. A more complete overview of the detector is given elsewhere [1]. The interaction region is surrounded by a 5-layer double-sided silicon vertex tracker (SVT) followed by a 40-layer drift chamber (DCH), which is filled with a mixture of isobutane and helium. The tracking devices are embedded in a 1.5-T solenoid field. The SVT and DCH measure the energy loss $d E / d x$, which provides charged particle identification in the low momentum region $(p<0.7 \mathrm{GeV} / c)$. Higher energetic charged particles are identified by a Cherenkov detector refered as DIRC (Detector of Internally Reflected Cherenkov light), which was designed to measure the angle of Cherenkov photons emitted when a charged particle passes one of the bars of fused silica.

\section{Dalitz plot analysis of $D^{0} \rightarrow \bar{K}^{0} K^{+} K^{-}$}

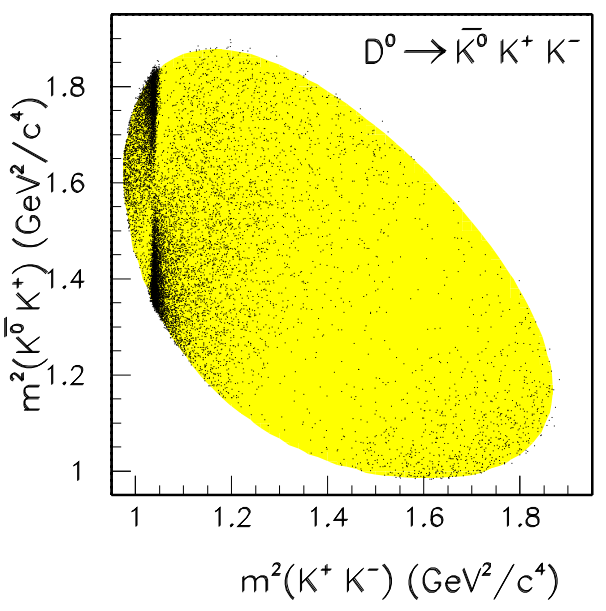

Figure 1: Dalitz plot of $D^{0} \rightarrow \bar{K}^{0} K^{+} K^{-}$

Dalitz plot analyses are useful in providing new information on resonances that contribute to threebody final states. They can help to enlighten the old puzzle of several light meson states, whose existence was reported by different experiments. In particular, the possible existence of broad states such as $\sigma(500)$ [2] and $\kappa(800)[3]$ were claimed by the E791 collaboration. These new evidences have reopened the discussion of the composition of the ground state $J^{P C}=0^{++}$ nonet, and of the possiblity that states like $a_{0}(980)$ or $f_{0}(980)$ may be 4-quark states [4]. This assumption can be tested by accurate measurements of branching fractions and couplings.

Fig. 1 shows the Dalitz plot of $D^{0} \rightarrow \bar{K}^{0} K^{+} K^{-}$. In order to measure the branching ratio $B R$ of $D^{0} \rightarrow$ $\bar{K}^{0} K^{+} K^{-}$and $D^{0} \rightarrow \bar{K}^{0} \pi^{+} \pi^{-}$the Dalitz plane was devided into approximately 500 cells and under usage of the efficiency corrected yields we determined

$$
B R=\frac{\Gamma\left(D^{0} \rightarrow \bar{K}^{0} K^{+} K^{-}\right)}{\Gamma\left(D^{0} \rightarrow \bar{K}^{0} \pi^{+} \pi^{-}\right)}=(15.8 \pm 0.1(\text { stat } .) \pm 0.5(\text { syst } .)) \times 10^{-2}
$$

A partial wave analysis has been done and we find a strong interference of the S- and Pwave amplitude contributions within the $\phi(1020)$ mass region. Under the assumption that the 
$\mathrm{P}$ contribution is entirely due to the $\phi(1020)$ the $a_{0}(980) \rightarrow K K$ lineshape has been determined almost background free. Furthermore an unbinned maximum likelihood fit has been performed in order to determine relative phases and amplitudes of intermediate resonant and non-resonant states. Table 1 summarizes the fit results. The decay is dominated by $D^{0} \rightarrow \bar{K}^{0} a_{0}(980)^{0}, D^{0} \rightarrow$ $\bar{K}^{0} \phi(1020)$ and $D^{0} \rightarrow K^{-} a_{0}(980)^{+}$. The $f_{0}(980)$ and the doubly-cabbibo suppressed contribution $\left(D^{0} \rightarrow K^{+} a_{0}(980)^{-}\right)$are consistent with 0 . The remaining contribution is non-uniform and could be interpreted by a tail of a broad resonance, e.g. $f_{0}(1400)$. Since the coupling of the $f_{0}(980)$ to the $K K$ system is poorly known it requires more data to make statements about the properties of the $f_{0}(980)$ and the $a_{0}(980)$.

\begin{tabular}{llll}
\hline Final state & Amplitude & Phase (radians) & Fraction $(\%)$ \\
\hline $\bar{K}^{0} a_{0}(980)^{0}$ & 1. & 0. & $66.4 \pm 1.6 \pm 7.0$ \\
\hline $\bar{K}^{0} \phi(1020)$ & $0.437 \pm 0.006 \pm 0.060$ & $1.91 \pm 0.02 \pm 0.10$ & $45.9 \pm 0.7 \pm 0.7$ \\
\hline$K^{-} a_{0}(980)^{+}$ & $0.460 \pm 0.017 \pm 0.056$ & $3.59 \pm 0.05 \pm 0.20$ & $13.4 \pm 1.1 \pm 3.7$ \\
\hline $\bar{K}^{0} f_{0}(1400)$ & $0.435 \pm 0.033 \pm 0.162$ & $-2.63 \pm 0.10 \pm 0.71$ & $3.8 \pm 0.7 \pm 2.3$ \\
\hline $\bar{K}^{0} f_{0}(980)$ & & & $0.4 \pm 0.2 \pm 0.8$ \\
\hline$K^{+} a_{0}(980)^{-}$ & & & $0.8 \pm 0.3 \pm 0.8$ \\
\hline Sum & & & $130.7 \pm 2.2$ \\
\hline
\end{tabular}

Table 1: Results from the Dalitz plot analysis of $D^{0} \rightarrow \bar{K}^{0} K^{+} K^{-}$

\section{Precise measurement of the $\Lambda_{c}^{+}$baryon mass}

So far the best $\Lambda_{c}^{+}$mass measurement was done by the CLEO collaboration using a sample of approximately 1000 events [5]. BABAR collected a very large sample of charmed-hadron decays and

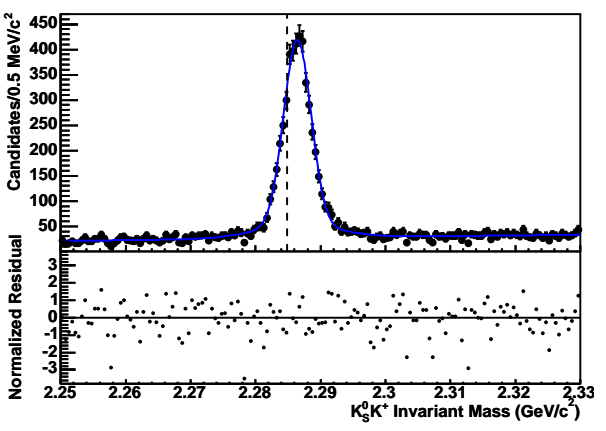

Figure 2: Invariant $\Lambda K_{S}^{0} K^{+}$mass. The lower part of the figure shows the normalized fit residuals. The dashed line indicates the present PDG value. charged particle momenta are measured with very high precision. The challenge of such a precision mass measurement lies in the track reconstruction uncertainties, arising from energy-loss correction and the knowledge of the magnetic field. Such systematic uncertainties tend to increase with increasing $Q$, whereas $Q$ denotes the 4-momentum transfer. Therefore we used low- $Q$ modes to reconstruct the $\Lambda_{c}^{+}$, such as $\Lambda_{c}^{+} \rightarrow \Lambda K_{S}^{0} K^{+}$ and $\Lambda_{c}^{+} \rightarrow \Sigma^{0} K_{S}^{0} K^{+}$.

Fig. 2 shows the invariant $\Lambda K_{S}^{0} K^{+}$mass fitted to a sum of two Gaussians with a common mean for signal and a linear function for combinatorial background. The signal yield was determined to be $4627 \pm 84$ and the fitted peak position is $(2286.44 \pm 0.04) \mathrm{GeV} / c^{2}$.

Four control samples $\left(\Lambda \rightarrow p \pi^{-}, K_{S}^{0} \rightarrow \pi^{+} \pi^{-}, \Lambda_{c}^{+} \rightarrow p K^{-} \pi^{+}\right.$and $\left.\Lambda_{c}^{+} \rightarrow p K_{S}^{0}\right)$ have been used to study mass dependencies on the amount of material assumed for the energy-loss correction and the magnetic field. As a result it was found that the $\Lambda_{c}$ mass needs to be corrected for 
underestimated energy loss. The correction is calculated by increasing the SVT material by $20 \%$. We find $m_{\Lambda_{c}^{+}}\left(\Lambda K_{s}^{0} K^{+}\right)=(2286.501 \pm 0.042$ (stat. $) \pm 0.144($ syst. $\left.)\right) \mathrm{MeV} / c^{2}$ and $m_{\Lambda_{c}^{+}}\left(\Sigma^{0} K_{s}^{0} K^{+}\right)=$ $(2286.303 \pm 0.181$ (stat. $) \pm 0.126$ (syst. $)) \mathrm{MeV} / c^{2}$. We combine these results to $m_{\Lambda_{c}^{+}}=(2286.46 \pm$ $0.14) \mathrm{MeV} / c^{2}$ which is in agreement with the present PDG value but much more precise.

\section{Production and decays of $\Omega_{c}^{0}$ baryons}

$\Omega_{c}^{0}$ baryons are reconstructed via their decay into $\Omega^{-} \pi^{+}$. The resulting invariant mass spectra have been fitted in 11 bins of the c.m. momentum $p^{*}\left(\Omega_{c}^{0}\right)$. In Fig. 3 the uncorrected momentum spectrum can be seen and it shows a very clear two peak structure. The peak above $1.5 \mathrm{GeV} / c$ can be identified as $\Omega_{c}^{0}$ production in continuum events (The blue bands illustrate the MC continuum expectation). Since the peak at low momenta is not seen in data taken below the $B \bar{B}$ threshold this peak can be interpreted as the first evidence for $\Omega_{c}^{0}$ baryons produced in $B$ decays.

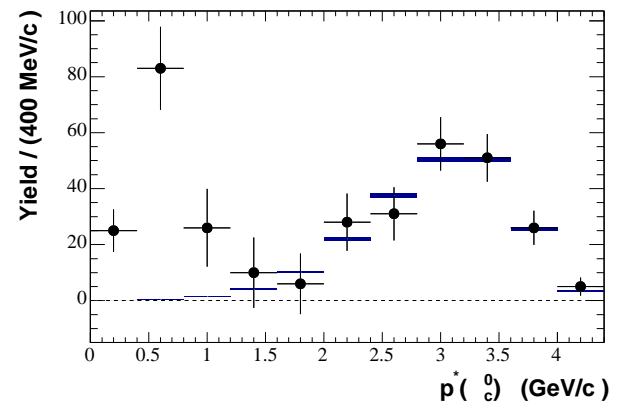

Figure 3: $\Omega_{c}^{0}$ momentum spectrum.

\section{Summary}

In summary a Dalitz plot analysis of $D^{0} \rightarrow \bar{K}^{0} K^{+} K^{-}$has been performed which indicates that this decay is dominated by $D^{0} \rightarrow \bar{K}^{0} a_{0}(980)^{0}, D^{0} \rightarrow \bar{K}^{0} \phi(1020)$ and $D^{0} \rightarrow K^{-} a_{0}(980)^{+}$. The $\Lambda_{c}^{+}$ mass has been measured with an inprovement in precision of factor 4 compared to the PDG value using low- $Q$-modes. We have found a first clear evidence for $\Omega_{c}$ production in $B$ meson decays.

\section{Aknowledgements}

We are grateful for the extraordinary contributions of our PEP-II colleagues in achieving the excellent luminosity and machine conditions that have made this work possible. The success of this project also relies critically on the expertise and dedication of the computing organizations that support BABAR. The collaborating institutions wish to thank SLAC for its support and the kind hospitality extended to them. This work is supported by the US Department of energy and national funding agencies.

\section{References}

[1] B. Aubert et al., Nucl. Instrum. Methods A479, 1 (2002)

[2] E. M. Aitala et al., Phys. Rev. Lett. 86, 770 (2001)

[3] E. M. Aitala et al., Phys. Rev. Lett. 89, 121801 (2002)

[4] F. E. Close and N. A. Tornquist, J. Phys. G28, R249

[5] P. Avery et al., Phys. Rev. D 43, 3599 